\title{
DESENVOLVIMENTO, CARACTERIZAÇÃO E APLICAÇÃO ELETROANALÍTICA DE UM COMPÓSITO FLUIDO DE ADESIVO EPÓXI, GRAFITE E CICLO-HEXANONA
}

\author{
Rodrigo Amorim Bezerra da Silva, Adriano César Rabelo, Otávio Luiz Bottecchia, Rodrigo Alejandro Abarza Muñoz e \\ Eduardo Mathias Richter* \\ Instituto de Química, Universidade Federal de Uberlândia, Av. João Naves de Ávila, 2121, 38400-902 Uberlândia - MG, Brasil
}

Recebido em 6/10/09; aceito em 25/1/10; publicado na web em 3/5/10

\begin{abstract}
DEVELOPMENT, CHARACTERIZATION AND ELECTROANALYTICAL APPLICATION OF A FLUID COMPOSITE MADE OF EPOXY ADHESIVE, GRAPHITE, AND CICLOHEXANONE. A fluid conducting composite material prepared from graphite powder, commercial epoxy resin Araldite ${ }^{\circledR}$, and cyclohexanone has been developed. The composition was optimized considering the mechanical properties as conductivity and adhesiveness using response surface methodology. This work employed cyclic voltammetry and amperometry to investigate the characteristics of such composite electrodes without and with the insertion of Prussian blue in the electrode body (bulk modified electrode). The composite electrodes were also successfully used for the amperometric detection of hydrogen peroxide at $0.0 \mathrm{~V} v s \mathrm{Ag} / \mathrm{AgCl}$.
\end{abstract}

Keywords: fluid graphite-epoxy composite; alternative fabrication method; Prussian blue modified electrode.

\section{INTRODUÇÃO}

O desenvolvimento de compósitos baseados em fases condutoras envolvidas por matrizes poliméricas, chamados de aglutinantes, tem conduzido a importantes avanços na química eletroanalítica. O compósito resulta da combinação de dois ou mais materiais que diferem na forma e na composição química e possui características físicas, mecânicas e químicas diferentes dos componentes iniciais. ${ }^{1}$ A propriedade elétrica do compósito depende, dentre vários aspectos, da natureza de cada componente, suas quantidades relativas e sua distribuição. A resistência elétrica é determinada pela conectividade das partículas condutoras no aglutinante, enquanto o último define a viscosidade do compósito. ${ }^{1}$

Vários trabalhos podem ser localizados sobre materiais condutores, como o carbono em suas várias formas alotrópicas e granulares, misturados a alguns polímeros orgânicos isolantes como o PVC,, 3 teflon, ${ }^{4,5}$ polietileno, ${ }^{6}$ poliestireno, ${ }^{7}$ nafion, ${ }^{8}$ acetato de celulose, ${ }^{9,10}$ adesivo epóxi, ${ }^{11,12}$ borracha silicone, ${ }^{13,14}$ dentre outros. Esses materiais podem ser utilizados, por exemplo, para construção de eletrodos para análises eletroquímicas e as vantagens citadas em relação a estes compósitos rígidos, de maneira geral, são facilidade de preparação e regeneração da superfície, resistência mecânica, durabilidade, relativo baixo custo de produção e possibilidade de inserção de modificadores no corpo do eletrodo e não somente em sua superfície. . $^{111,15,16}$

Atualmente existe um grande interesse em compósitos de grafite e adesivo epóxi (mistura de massas iguais de resina epóxi com endurecedor) para diversas finalidades analíticas, como na fabricação de eletrodos convencionais, ${ }^{17,18}$ dispositivos com arranjo de microeletrodos de grafite, ${ }^{15,19}$ suporte para a formação de filmes metálicos in situ, ${ }^{20,21}$ suporte para modificações da superfície na fabricação de biossensores, ${ }^{22}$ imunossensores, ${ }^{23}$ genossensores ${ }^{24,25}$ e dispositivos para análise de pesticidas. ${ }^{26}$ São encontrados também em arranjos para células microfluídicas ${ }^{27}$ e em microchips para detectores em eletroforese capilar. ${ }^{28}$

Na literatura, dois procedimentos básicos de preparação de compósitos rígidos de grafite são apresentados. No primeiro, o condutor e o aglutinante são misturados manualmente na ausência de solvente orgânico ${ }^{12,29}$ e no segundo, as duas fases são misturadas na presença

\footnotetext{
*e-mail: emrichter@iqufu.ufu.br
}

de um solvente orgânico, podendo então originar as tintas condutoras empregadas na fabricação de eletrodos impressos (screen-printed electrodes). ${ }^{30}$ Atualmente, tintas usando diferentes condutores e aglutinantes estão disponíveis no mercado. No entanto, informações a respeito da produção destas tintas são pouco divulgadas e geralmente se encontram sob proteção de patentes. ${ }^{31,32}$ Alguns poucos protocolos da fabricação de tintas elaboradas em laboratório, chamadas de homemade inks, podem ser localizados na literatura ${ }^{9,33}$ e consistem na mistura de acetato de celulose, ciclo-hexanona, acetona e pó de grafite.

Neste trabalho apresentamos um protocolo para a produção em laboratório de um compósito à base de adesivo epóxi e grafite na presença do solvente ciclo-hexanona (tinta condutora e adesiva). As proporções entre os três componentes da mistura foram avaliadas em relação a características como fluidez, adesividade e resistência ôhmica. Após a otimização do compósito, algumas características como o tempo de armazenamento do compósito pré e pós-cura, reprodutibilidade intereletrodo e espessura do compósito quando usado como eletrodo foram avaliadas. Além disso, a resposta eletroquímica do compósito foi comparada a outros materiais (carbono vítreo e pasta de carbono) e sua potencialidade foi avaliada como sensor para peróxido de hidrogênio, após a incorporação do modificador químico azul da Prússia no corpo do eletrodo.

\section{PARTE EXPERIMENTAL}

\section{Reagentes e soluções}

As soluções foram preparadas utilizando água desionizada do sistema de purificação Milli-Q Plus da Millipore (resistividade $c a 18 \mathrm{~m} \Omega \mathrm{cm}$ ). Os reagentes utilizados eram todos de grau analítico. O adesivo epóxi Araldite $^{\circledR}(24 \mathrm{~h})$, do fabricante Brascola, foi adquirido em mercado local. O pó de grafite foi adquirido da empresa Sigma-Aldrich (granulometria ca $2 \mu \mathrm{m}$ ). A modificação química do pó de grafite com azul da Prússia foi efetuada através do seguinte procedimento: grafite foi adicionado sob agitação (por $10 \mathrm{~min}$ ) a uma mistura equimolar de cloreto férrico e ferricianeto de potássio em meio de ácido clorídrico. Informações mais detalhadas podem ser localizadas em procedimento descrito por Moscone e colaboradores. ${ }^{34}$ A cola de prata (PC 9045) foi adquirida da empresa Joint Metal Comércio Ltda (Diadema/SP). 


\section{Instrumentação}

Os estudos eletroquímicos foram realizados em célula de acrílico com volume de $5 \mathrm{~mL}$ usando um eletrodo de $\mathrm{Ag} / \mathrm{AgCl} / \mathrm{KCl}$ sat. construído em laboratório ${ }^{35}$ e platina como eletrodos de referência e auxiliar, respectivamente. Estudos comparativos de desempenho foram realizados usando carbono vítreo $(\phi=3,0 \mathrm{~mm}$, Metrohm $)$ e pasta de carbono $(\phi=2,0 \mathrm{~mm})$ como eletrodos de trabalho. Os experimentos voltamétricos foram realizados usando um potenciostato Micro Autolab III (Autolab/Eco Chemie) interfaceado a um computador com o software GPES 4.9.007 para a aquisição dos dados.

\section{Procedimento de produção do compósito}

Após estudos de otimização para a preparação dos compósitos condutores de grafite, o seguinte procedimento foi adotado: (1) 0,10 $\mathrm{g}$ de resina epóxi (Araldite ${ }^{\circledR} 24 \mathrm{~h}$, Brascola) foram adicionadas a 3,0 mL de ciclo-hexanona sob agitação magnética por $5 \mathrm{~min}$, para a dissolução da resina no solvente; (2) adição de $0,10 \mathrm{~g}$ de poliaminoamida (endurecedor), mantendo-se a agitação por mais $10 \mathrm{~min}$, para a dissolução do endurecedor no solvente; (3) adição lenta de 2,0 g de grafite (granulometria até $2 \mu \mathrm{m}$ ), mantendo-se o sistema sob agitação por $24 \mathrm{~h}$.

Após esta homogeneização, a mistura apresenta boa fluidez por um período superior a 30 dias, desde que mantida em frasco hermeticamente fechado. Durante este tempo, o material pode ser utilizado para a produção de eletrodos transferindo-se (por pincelamento) para a superfície de interesse. Para sua cura, o compósito deve ser mantido à temperatura ambiente por $24 \mathrm{~h}$. Recomenda-se o uso deste compósito em até 5 semanas após o seu preparo, para evitar uma polimerização in situ (no fundo do frasco de armazenagem) e a secagem do solvente. É importante lembrar que a tinta condutora sempre deve ser agitada por alguns minutos antes do uso (para restabelecer a homogeneidade da mistura)

\section{Otimização da composição do compósito}

A otimização das proporções entre as frações mássicas dos componentes adesivo epóxi, grafite e ciclo-hexanona foi realizada em função da resistência elétrica do material. Estas medidas foram obtidas da seguinte maneira: (1) inicialmente, 13 compósitos com diferentes proporções entre adesivo epóxi, grafite e ciclo-hexanona foram produzidos (Figura 1A); (2) em seguida, cada proporção foi pincelada sobre bandas geminadas de cobre (espaçadas entre si em $0,6 \mathrm{~mm}$ ) previamente produzidas sobre uma placa de circuito impresso. Após a cura (evaporação do solvente e fim da polimerização residual) por 24 h, a resistência de cada compósito foi medida usando um multímetro. Estas medidas foram realizadas em quadruplicata. (3) A partir das medidas de resistência média e das dimensões das placas de cobre, a condutividade $\left(\sigma \mathrm{m}^{-1}\right)$ de cada compósito foi obtida através da equação da segunda lei de $\mathrm{Ohm}(\sigma=L A / R)$; (4) os treze pontos do planejamento de misturas com os respectivos resultados experimentais de condutividade foram inseridos em um programa estatístico (Statistica 6.0 - StatSoft Inc. Tulsa, OK, EUA) e obtevese uma equação e uma superfície de resposta em função da fração mássica de cada componente.

\section{Construção dos eletrodos de compósito}

Os eletrodos de trabalho à base de compósito de grafite foram construídos através do seguinte procedimento (Figura 1S, material suplementar): (1) com o auxílio de um polímero termo moldável, um fio de cobre $(\phi \approx 1,5 \mathrm{~mm})$ foi fixado no interior de uma ponteira de micropipeta de polietileno (10-100 $\mu \mathrm{L}$ ), a $3 \mathrm{~mm}$ da extremidade de menor abertura, de modo a produzir uma pequena cavidade. Antes, porém, as paredes internas da extremidade da ponteira foram recobertas com uma película fina de adesivo epóxi Araldite ${ }^{\circledR} 24$ h; (2) em seguida o compósito, ainda fluido, é facilmente introduzido na cavidade com auxílio de um fino bastão inerte (agulha de aço inoxidável, por exemplo). O filme de adesivo epóxi sobre a parede interna da ponta da ponteira evita o contato direto entre o compósito condutor e o polietileno, devido a pouca aderência. (3) Após o tempo de cura (24 h), a ponteira contendo o compósito foi sucessivamente polida com lixas d'água de granulação 400, 600 e 1000, respectivamente, na presença de água desionizada. O polimento foi interrompido quando se tem uma superfície sem degraus, ou seja, com o compósito no mesmo nível da ponteira, o que é conveniente para uso como eletrodo. Como as lixas mais grossas (400 e 600) são capazes de desgastar o polietileno da ponteira, pode-se ajustar a espessura do compósito em relação ao contato elétrico (cobre). As medidas da espessura do compósito (interface compósito/solução e compósito/fio de cobre) foram efetuadas com um paquímetro. O condicionamento eletroquímico dos eletrodos compósitos (previamente ao uso) foi realizado usando voltametria cíclica $\left(50 \mathrm{mV} \mathrm{s}^{-1}\right)$ com varreduras sucessivas em ácido sulfúrico $0,10 \mathrm{~mol} \mathrm{~L} \mathrm{~L}^{-1}$ entre $-0,2 \mathrm{e}+0,7 \mathrm{~V}$ até a obtenção de voltamogramas com perfis semelhantes (estabilização do sinal).

\section{RESULTADOS E DISCUSSÃO}

\section{Otimização da composição do compósito}

Na Figura 1A, junto aos pontos que representam as condições investigadas, encontram-se as respectivas medidas de condutividade ( $n=4$, com desvios padrões relativos entre 5 e 15\%). A Figura 1B apresenta a superfície de resposta da condutividade em função da fração mássica de cada componente do compósito e a Equação 1 representa matematicamente esta superfície.

$\sigma\left(S m^{-1}\right)=-32,87 A E+257,66 G R-20,76 C H-529,47(A E x G R)$

$-58,87(A E x C H)+63,09(G R \times C H)$

em que: $\sigma\left(\mathrm{S} \mathrm{m}^{-1}\right)$ é a condutividade em Siemens por metro; $A E$ é a fração mássica do adesivo epóxi (resina epóxi com poliaminoamida); $G R$ é a fração mássica de grafite; $C H$ é a fração mássica de ciclo-hexanona.

Como pode ser observado na Equação 1, os efeitos individuais dos componentes adesivo epóxi, poliaminoamida e ciclo-hexanona apresentam uma influência negativa na condutividade da mistura, pois o polímero de adesivo epóxi é um isolante elétrico ${ }^{1}$ e o solvente, conforme descrito na literatura, pode deixar espaços vazios após a sua volatilização, piorando a interação entre as partículas condutoras de grafite. ${ }^{9}$ Dentre os solventes estudados (tetra-hidrofurano e ciclohexanona), a ciclo-hexanona apresentou os melhores resultados (menos volátil, menos tóxica e odor menos desagradável), pois devido sua menor velocidade de evaporação, a dimensão média dos espaços deixados deve ser menor, o que resultou em uma superfície mais suavizada. ${ }^{9} \mathrm{O}$ grafite é o único componente que possui um efeito positivo na condutividade elétrica, pois é um semicondutor com condutividade consideravelmente elevada. ${ }^{36}$

Diferentes características foram observadas para os diversos compósitos construídos. Os compósitos com grande porcentagem de adesivo epóxi eram rígidos, mas pouco condutores ou isolantes. Os compósitos com alta porcentagem de grafite são facilmente quebráveis, pouco adesivos sobre superfícies e pouco condutores devido à baixa interação entre as partículas. O ponto $x$ da Figura $1 \mathrm{~A}$ é uma mistura planejada onde não foi possível medir a resistência, devido à 




Figura 1. (A) Proporções estudadas entre os três constituintes do compósito (grafite, Araldite ${ }^{\circledast}$ e ciclo-hexanona) com as respectivas condutividades ( $S$ $\left.m^{-1}\right) ;(B)$ superfície de resposta da condutividade dos compósitos em função da fração mássica de grafite, adesivo epóxi Araldite ${ }^{\circledast}$ e solvente ciclo-hexanona

baixa adesividade dessa mistura nas superfícies de cobre e fenolite da placa de circuito impresso. Já os compósitos com alta porcentagem de solvente possuem excelente fluidez, mas pouca rigidez, após a cura.

Os resultados apresentados na Figura 1 foram usados para selecionar um compósito que aliasse as propriedades condutoras do grafite, as adesivas do adesivo epóxi e com fluidez razoável para facilitar o processo de construção de microdispositivos com desempenho eletroquímico adequado. Essas condições são satisfeitas na proporção 0,04/0,4/0,56 (m/m) de adesivo epóxi/grafite/ciclo-hexanona, respectivamente. Nesta composição, o compósito, após a cura, apresenta uma condutividade de $57 \mathrm{~S} \mathrm{~m}^{-1}$.

A inclusão da ciclo-hexanona na produção do compósito de grafite gerou um material fluido e estável se mantido em frasco hermeticamente fechado. Esta característica é interessante, pois o material pode ser armazenado e os eletrodos poderão ser construídos conforme a necessidade e com a composição idêntica aos anteriores. As respostas eletroquímicas (ferricianeto de potássio $1 \mathrm{mmol} \mathrm{L}^{-1} \mathrm{em}$ meio de $\mathrm{KCl}$ 0,1 $\mathrm{mol} \mathrm{L}^{-1}$ ) dos eletrodos construídos com o compósito proposto no mesmo dia de sua produção ou após 5 semanas de armazenado em frasco fechado foram bastante similares $\left(\mathrm{DPR}_{\mathrm{ipc}}=5,0 \% ; \mathrm{n}=5\right)$. Neste período, observou-se uma queda de $20 \%$ na condutividade do compósito. No entanto, esta variação não comprometeu os resultados obtidos. Além disso, os eletrodos produzidos com este compósito (após a cura) apresentaram o mesmo perfil eletroquímico de resposta por um período de 6 meses (tempo estudado), desde que seja utilizado em sua faixa de trabalho, o que depende do eletrólito utilizado. Antes do reuso do eletrodo, uma limpeza é necessária, conforme descrito no procedimento experimental.

A capacidade adesiva do compósito está diretamente relacionada à porcentagem do polímero presente na mistura. Porém, a presença do polímero é inversamente proporcional à condutividade do material. Como um dos objetivos do trabalho era a produção de um compósito com boa capacidade adesiva (útil também como cola condutora), estudos para atenuar os efeitos da resistência do material no desempenho como sensor foram efetuados. A Figura 2 apresenta os voltamogramas cíclicos de ferricianeto de potássio $5,0 \mathrm{mmol} \mathrm{L} \mathrm{L}^{-1}$ em meio de $\mathrm{KCl}$ 0,5 mol L-1 obtidos para eletrodos do compósito otimizado em diferentes espessuras $(5,3$ e $1 \mathrm{~mm})$.

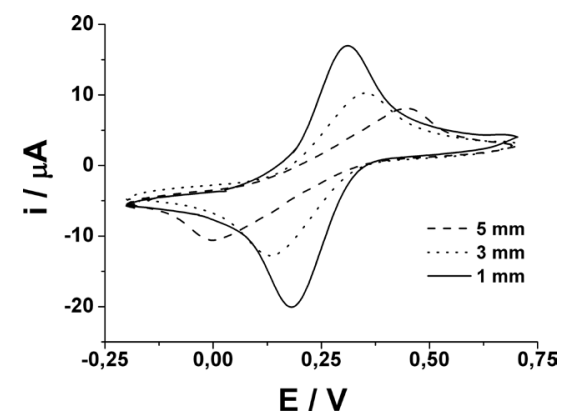

Figura 2. Voltamogramas cíclicos registrados em uma solução de ferricianeto de potássio 5,0 $\mathrm{mmol} \mathrm{L}^{-1}$ em eletrólito suporte de $\mathrm{KCl}$ 0,5 $\mathrm{mol} \mathrm{L}^{-1} \mathrm{como}$ microdispositivo em diferentes espessuras da camada de compósito de grafite

Como pode ser observado, numa espessura de $1 \mathrm{~mm}$ entre solução e fio de cobre, existe uma menor diferença entre os potenciais de pico catódico e anódico ( $\Delta \mathrm{Ep})$, além de um aumento nos valores de corrente. Isto indica que a resistência elétrica do eletrodo de trabalho diminui em função da diminuição da espessura do compósito, o que facilita a transferência eletrônica entre as espécies eletroativas e o eletrodo.

Uma vantagem que este procedimento de preparação do compósito oferece está relacionada com a homogeneidade na mistura de seus constituintes. Em 5 eletrodos de trabalho diferentes construídos usando o procedimento proposto, boa reprodutibilidade nas medidas eletroquímicas de ferricianeto de potássio $1 \mathrm{mmol} \mathrm{L}^{-1} \mathrm{em}$ meio de $\mathrm{KCl}$ $0,1 \mathrm{~mol} \mathrm{~L}^{-1}$ foi observada $\left(\mathrm{DPR}_{\mathrm{ipc}}=4,0 \% ; \mathrm{n}=5\right)$. Outra vantagem apresentada por este compósito de grafite está relacionada a não necessidade de se utilizarem as dispendiosas colas de prata comerciais para promover a adesão do material ao fio de cobre (contato elétrico), comum na maioria dos trabalhos sobre este tema., ${ }^{9,1929}$ Estudos comparativos demonstraram que o perfil de voltamogramas cíclicos obtidos é idêntico, independentemente da forma do contato elétrico entre o fio de cobre e o compósito (com ou sem cola de prata). ${ }^{37}$

Na Figura 3 são apresentados voltamogramas cíclicos comparativos de ferrocianeto de potássio $5,0 \mathrm{mmol} \mathrm{L}^{-1}$ em meio de $\mathrm{KCl}$ $0,5 \mathrm{~mol} \mathrm{~L}^{-1}$ entre os eletrodos de compósito, carbono vítreo e pasta de carbono. Vale ressaltar que na Figura 3 as correntes obtidas nos voltamogramas são normalizadas pela área geométrica de cada eletrodo, sendo o resultado então expresso em função da densidade de corrente (j). O eletrodo de compósito de grafite e o eletrodo de carbono vítreo tiveram resultados semelhantes em relação à separação entre os potenciais de pico anódico e catódico $\left(\Delta \mathrm{E}_{\text {pico }} \sim 80 \mathrm{mV}\right)$. Estes resultados mostram uma menor resistência à transferência de carga do eletrodo de compósito em relação ao de pasta de carbono $\left(\Delta \mathrm{E}_{\text {pico }}\right.$ $\sim 180 \mathrm{mV}$ ). A diferença entre as densidades de corrente está diretamente relacionada com a área efetiva de cada eletrodo de trabalho.

Um tema recentemente explorado na construção de eletrodos à base de compósitos é sua impregnação com nanopartículas de diferentes materiais como ouro, platina, bismuto e azul da Prússia, entre outros. ${ }^{34,37-39}$ A inserção destes materiais tem como objetivo trazer características interessantes aos compósitos, como aumento da área superficial dos modificadores, economia de materiais dispendiosos (metais nobres) e maior seletividade para análise de diversas espécies químicas. Dentro deste contexto, estudos para modificação do compósito de grafite proposto com partículas de azul da Prússia foram realizados. Inicialmente, pó de grafite foi modificado com partículas de azul da Prússia conforme descrito na literatura. ${ }^{34}$ Para a produção do eletrodo modificado, a proporção entre adesivo epóxi/grafite/ciclo-hexanona foi 


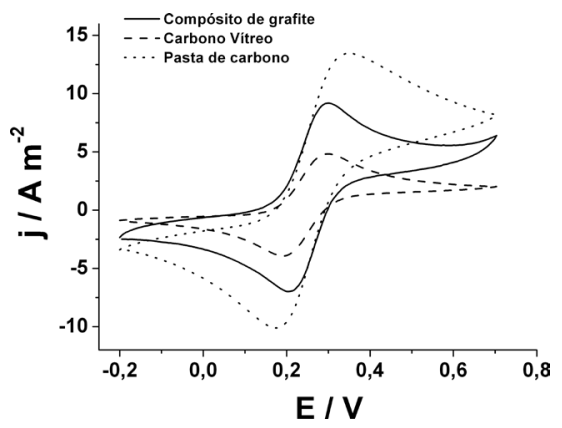

Figura 3. Voltamogramas cíclicos obtidos (em função da densidade de corrente, j) em uma solução de ferrocianeto de potássio $5 \mathrm{mmol} \mathrm{L}^{-1}$ em eletrólito suporte de $\mathrm{KCl}$ 0,5 $\mathrm{mol} \mathrm{L}^{-1}$ em eletrodos de trabalho de compósito de grafite, carbono vítreo ou pasta de carbono

mantida em 0,04/0,4/0,56 (m/m), respectivamente. Na fração grafite $(0,4)$, o melhor desempenho foi obtido para um mistura de 10 e $90 \%$ de grafite modificado e puro (em massa), respectivamente. Após a cura e limpeza mecânica do compósito (imobilizado em ponteira de micropipeta), um eletrodo de compósito modificado com partículas de azul da Prússia foi obtido. O comportamento voltamétrico em meio de $\mathrm{KCl}$ 0,10 $\mathrm{mol} \mathrm{L}^{-1}$ do eletrodo de compósito modificado em relação ao eletrodo não modificado é apresentado na Figura 4A. Com base neste voltamograma, o excesso superficial $(\Gamma=\mathrm{Q} / \mathrm{nFA})$ de partículas de azul da Prússia foi calculado em 4,0 x 10-8 mol por $\mathrm{m}^{2}$ de grafite. No eletrodo modificado com azul da Prússia, picos característicos de redução $(0,14 \mathrm{~V})$ e oxidação $(0,20 \mathrm{~V})$ do par $\mathrm{Fe}(\mathrm{II}) / \mathrm{Fe}(\mathrm{III})$ podem ser observados. A transferência eletrônica no compósito modificado proposto neste trabalho é favorecida $(\Delta \mathrm{Ep}=60 \mathrm{mV})$ em relação a outros anteriormente publicados usando este modificador em compósitos, como o trabalho de Moscone et al. ${ }^{34}(\Delta \mathrm{Ep}=140 \mathrm{mV})$ e O'Halloran et $a l .{ }^{40}(\Delta \mathrm{Ep}=110 \mathrm{mV})$. Na Figura 4B é apresentado um cronoamperograma com aplicação de $0,0 \mathrm{~V}$ vs $\mathrm{Ag} / \mathrm{AgCl}$ ao eletrodo de compósito modificado com partículas de azul da Prússia após sucessivas adições de $10 \mu \mathrm{mol} \mathrm{L}^{-1}$ de peróxido de hidrogênio. Observa-se uma curva linear até $90 \mu \mathrm{mol} \mathrm{L}^{-1}$ com um coeficiente de correlação igual a 0,995 e sensibilidade de $4 \mathrm{nA} \mathrm{L} / \mu \mathrm{mol} \mathrm{H}_{2} \mathrm{O}_{2}$ (Figura $4 \mathrm{C}$ ). A reprodutibilidade dos eletrodos modificados com azul da Prússia é dependente do processo de construção, pois as ponteiras utilizadas apresentam formato cônico, o que dificulta a obtenção de áreas geométricas idênticas. Este efeito é mais pronunciado do que a falta de homogeneidade do compósito.

Segundo a literatura, ${ }^{34}$ o transdutor que contém azul da Prússia incorporado ao corpo do eletrodo apresenta algumas vantagens frente a eletrodos com filmes de azul da Prússia eletroquimicamente depositados, como sensibilidade superior, menor tempo de resposta, maior reprodutibilidade, maior estabilidade operacional em diferentes meios e possibilidade de obtenção de uma superfície renovada após polimento (caso haja contaminação ou desgaste), pois o compósito atua como um reservatório de azul da Prússia.

\section{CONCLUSÕES}

O compósito produzido a partir de adesivo epóxi, grafite e ciclohexanona apresenta-se como material alternativo e de baixo custo para a construção de eletrodos de trabalho com potencialidade em diversas aplicações. A inclusão do solvente na produção do compósito gerou um material com boa fluidez, o que permite a produção de eletrodos com excelente homogeneidade, pois é possível o uso de agitação magnética na mistura dos componentes (boa reprodutibilidade intereletrodos). Além disto, o solvente permite a utilização do mesmo compósito por 1 mês para a construção de eletrodos, desde que a mistura seja mantida em frasco hermeticamente fechado. Adicionalmente, foi demonstrada


Figura 4. (A) Voltamogramas em $\mathrm{KCl}$ 0,10 $\mathrm{mol} \mathrm{L}^{-1}$ com eletrodos de trabalho de compósito de grafite puro (linha tracejada) e compósito de grafite modificado com partículas de azul da Prússia (linha contínua); (B) cronoamperograma para adições sucessivas de $10 \mu \mathrm{mol} L^{-1}$ de peróxido de hidrogênio utilizando-se o eletrodo de trabalho de compósito modificado com partículas de azul da Prússia; (C) curva de calibração obtida para os resultados apresentados em (B). Velocidade de varredura em (A): $20 \mathrm{mV} \mathrm{s}^{-1}$; Potencial aplicado em (B): 0,0 V (vs Ag/AgCl/KCl sat.)

a potencialidade do compósito na incorporação de modificadores no corpo do eletrodo. Partículas de azul da Prússia foram inseridas com sucesso no compósito, com bom desempenho na análise de peróxido de hidrogênio no potencial de $0,0 \mathrm{~V} v s \mathrm{Ag} / \mathrm{AgCl}$. Além disto, as características peculiares deste material permitiram a inserção de eletrodos à base de compósito de grafite no interior de células microfluídicas, conforme artigo recentemente publicado. ${ }^{27}$

\section{MATERIAL SUPLEMENTAR}

A Figura 1S está disponível, em http://quimicanova.sbq.org.br, na forma de arquivo PDF, com acesso livre.

\section{AGRADECIMENTOS}

À CAPES, FAPEMIG e ao CNPq pelo apoio financeiro. R. A. B. da Silva agradece ao CNPq pela bolsa de doutorado concedida (141972/2009-2).

\section{REFERÊNCIAS}

1. Cespedes, F.; MartinezFabregas, E.; Alegret, S.; TrAC, Trends Anal. Chem. 1996, 15, 296. 
2. Albertus, F.; Llerena, A.; Alpizar, J.; Cerda, V.; Luque, M.; Rios, A.; Valcarcel, M.; Anal. Chim. Acta 1997, 355, 23.

3. Aguilar, R.; Davila, M. M.; Elizalde, M. P.; Mattusch, J.; Wennrich, R.; Electrochim. Acta 2004, 49, 851.

4. Klatt, L. N.; Connell, D. R.; Adams, R. E.; Honigberg, I. L.; Price, J. C.; Anal. Chem. 1975, 47, 2470.

5. Pena, N.; Ruiz, G.; Reviejo, A. J.; Pingarron, J. M.; Anal. Chem. 2001, 73, 1190 .

6. Armentrout, D. N.; McLean, J. D.; Long, M. W.; Anal. Chem. 1979, 51, 1039.

7. Park, J.; Shaw, B. R.; Anal. Chem. 1989, 61, 848.

8. Barroso-Fernandez, B.; Lee-Alvarez, M. T.; Seliskar, C. J.; Heineman, W. R.; Anal. Chim. Acta 1998, 370, 221.

9. Barsan, M. M.; Pinto, E. M.; Florescu, M.; Brett, C. M. A.; Anal. Chim. Acta 2009, 635, 71

10. Wring, S. A.; Hart, J. P.; Bracey, L.; Birch, B. J.; Anal. Chim. Acta 1990, 231, 203.

11. Ramirez-Garcia, S.; Alegret, S.; Cespedes, F.; Forster, R. J.; Anal. Chem. 2004, 76, 503 .

12. Moreno-Baron, L.; Merkoci, A.; Alegret, S.; Electrochim. Acta 2003, 48, 2599.

13. de Oliveira, A. C.; dos Santos, S. X.; Cavalheiro, E. T. G.; Talanta 2008, $74,1043$.

14. dos Santos, S. X.; Mazo, L. H.; Cavalheiro, E. T. G.; J. Braz. Chem. Soc. 2008, 19, 1600.

15. Ramirez- Garcia, S.; Alegret, S.; Cespedes, F.; Forster, R. J.; Analyst 2002, 127, 1512.

16. Kirgoz, U. A.; Odaci, D.; Timur, S.; Merkoci, A.; Pazarlioglu, N.; Telefoncu, A.; Alegret, S.; Bioelectrochemistry 2006, 69, 128.

17. Calixto, C. M. F.; Dissertação de Mestrado, Universidade Federal de São Carlos, Brasil, 2008.

18. Skvortsova, L. I.; Kiryushov, V. N.; Aleksandrova, T. P.; Karunina, O. V.; J. Anal. Chem. 2008, 63, 258.

19. O'Hare, D.; Macpherson, J. V.; Willows, A.; Electrochem. Commun. 2002, 4, 245.
20. Kirgoz, U. A.; Marin, S.; Pumera, M.; Merkoci, A.; Alegret, S.; Electroanalysis 2005, 17, 881.

21. Carregalo, S.; Merkoci, A.; Alegret, S.; Microchim. Acta 2004, 147, 245.

22. Kirgoz, U. A.; Odaci, D.; Timur, S.; Merkoci, A.; Alegret, S.; Besun, N.; Telefoncu, A.; Anal. Chim. Acta 2006, 570, 165.

23. Pividori, M. I.; Lermo, A.; Bonanni, A.; Alegret, S.; del Valle, M.; Anal. Biochem. 2009, 388, 229.

24. Pividori, M. I.; Alegret, S.; Anal. Lett. 2003, 36, 1669.

25. Pividori, M. I.; Merkoci, A.; Alegret, S.; Biosens. Bioelectron. 2003, 19 , 473.

26. Manea, F.; Radovan, C.; Corb, I.; Pop, A.; Burtica, G.; Malchev, P.; Picken, S.; Schoonman, J.; Electroanalysis 2008, 20, 1719.

27. da Silva, R. A. B.; de Almeida, E. G. N.; Rabelo, A. C.; da Silva, A. T. C.; Ferrreira, L. F.; Richter, E. M.; J. Braz. Chem. Soc. 2009, 20, 1235.

28. Pumera, M.; Merkoci, A.; Alegret, S.; Electroanalysis 2006, 18, 207.

29. Calixto, C. M. F.; Cervini, P.; Cavalheiro, E. T. G.; Quim. Nova 2008 , 31,2194

30. Nascimento, V. B.; Angnes, L.; Quim. Nova 1998, 21, 614.

31. Krishnamurthi, S.; Rao, V. K.; Sharma, M. K.; Singh, B.; Srinivasan, S.; India Defense Res\&Dev Org, IN200701669-I1, 2009.

32. Su, C.; Cheng, W.; Hung, M.; Wu, T.; Su, C; Cheng, W; Hung, M; Wu, T.; US2008160543-A1, 2008

33. Wring, S. A.; Hart, J. P.; Analyst 1992, 117, 1281.

34. Moscone, D.; D’Ottavi, D.; Compagnone, D.; Palleschi, G.; Amine, A.; Anal. Chem. 2001, 73, 2529.

35. Pedrotti, J. J.; Angnes, L.; Gutz, I. G. R.; Electroanalysis 1996, 8, 673. 36. Horton, G. K.; Tauber, G. E.; Phys. Rev. 1954, 93, 911.

37. da Silva, R. A. B.; Dissertação de Mestrado, Universidade Federal de Uberlândia, Brasil, 2009.

38. Dai, X.; Wildgoose, G. G.; Salter, C.; Crossley, A.; Compton, R. G.; Anal. Chem. 2006, 78, 6102.

39. Newman, J. D.; White, S. F.; Tothill, I. E.; Turner, A. P. F.; Anal. Chem. 1995, 67, 4594

40. O’Halloran, M. P.; Pravda, M.; Guilbault, G. G.; Talanta 2001, 55, 605. 


\section{DESENVOLVIMENTO, CARACTERIZAÇÃO E APLICAÇÃO ELETROANALÍTICA DE UM COMPÓSITO}

\section{FLUIDO DE ADESIVO EPÓXI, GRAFITE E CICLO-HEXANONA}

Rodrigo Amorim Bezerra da Silva, Adriano César Rabelo, Otávio Luiz Bottecchia, Rodrigo Alejandro Abarza Muñoz e Eduardo Mathias Richter*

Instituto de Química, Universidade Federal de Uberlândia, Av. João Naves de Ávila, 2121, 38400-902 Uberlândia - MG, Brasil

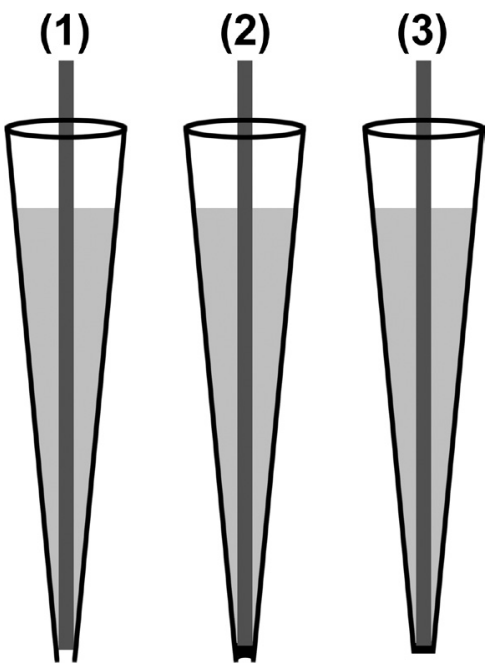

Figura 1S. Etapas de construção dos eletrodos à base de compósito de grafite. (1) Fixação de um fio de cobre ( $\phi \approx 1,5$ mm) no interior de uma ponteira de micropipeta de polietileno $(10-100 \mu \mathrm{L})$; (2) introdução do compósito, ainda fluido, na cavidade inferior; (3) após o tempo de cura (24 h), polimento do conjunto até que o compósito apresente o mesmo nível da ponteira 